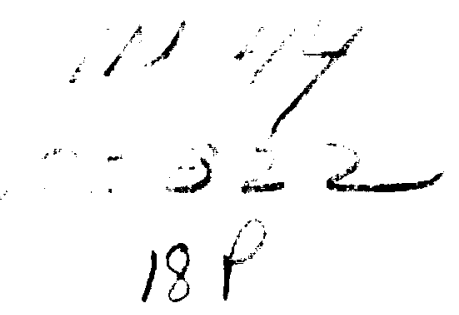

\title{
Emittance Theory for Thin Film Selective Emitter
}

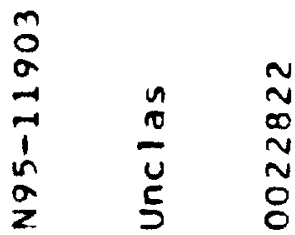

Donald L. Chubb

Lewis Research Center

Cleveland, Ohio

Roland A. Lowe

Kent State University

Kent, Ohio

Brian S. Good

Lewis Research Center

Cleveland, Ohio

Prepared for the

First Conference on Thermophotovoltaic Generation of Electricity sponsored by the National Renewable Energy Laboratory

Copper Mountain, Colorado, July 24-26, 1994 



\title{
Emittance Theory for Thin Film Selective Emitter
}

\author{
Donald L. Chubb*, Roland A. Lowe ${ }^{+}$and Brian S. Good* \\ *NASA Lewis Research Center, Cleve., Ohio 44135 \\ ${ }^{+}$School of Technology, Kent State Univ., Kent, Ohio 44242
}

\begin{abstract}
Thin films of high temperature garnet materials such as yttrium aluminum garnet (YAG) doped with rare earths are currently being investigated as selective emitters. This paper presents a radiative transfer analysis of the thin film emitter. From this analysis the emitter efficiency and power density are calculated. Results based on measured extinction coefficients for erbium-YAG and holmium-YAG are presented. These results indicate that emitter efficiencies of $50 \%$ and power densities of several watts/cm 2 are attainable at moderate temperatures $(<1750 \mathrm{~K})$.
\end{abstract}

\section{INTRODUCTION}

There are two emitter designs that can be used in a thermophotovoltaic (TPV) energy conversion system. In a selective emitter system most of the emitted photons have energy greater than the bandgap energy of the photovoltaic (PV) cells. In a thermal emitter system a narrow bandpass filter is used to shape the emission spectrum. The ideal filter allows all photons within the band to pass from emitter to the PV cells and reflects all photons outside the band back to the emitter. Both the selective emitter system and the filter system are feasible. In this paper we present the radiative transfer theory necessary to calculate the spectral emittance of a thin film selective emitter. From the spectral emittance, the emitter efficiency is calculated.

The solid state selective emitters of most interest are compounds containing the rare earths. For doubly and triply charged ions of these elements in crystals, the orbits of the valence $4 \mathrm{f}$ electrons, which account for most of the emission and absorption, lie inside the $5 \mathrm{~s}$ and 5 p electron orbits. As a result, the rare earth ions in the solidstate have radiative characteristics much like they would have if they were isolated. They emit in narrow bands rather than in a continuum as do most solids. The $5 \mathrm{~s}$ and $5 \mathrm{p}$ electrons "shield" the $4 \mathrm{f}$ valence electrons from the surrounding ions in the crystal. The spectra of these rare earth ions in crystals have been extensively studied. Most of this work is summarized in the text of Dieke(1).

Early spectral emittance work(2) on rare earth oxides showed strong emission bands. However, the emittance for photon energies below the bandgap for PV materials was also significant. As a result, the efficiency of these emitters was low. In the last few years, however, large improvement in rare earth emitters has occurred. The Welsbach mantle type emitters of Nelson and Parent $(3,4)$ have a single strong emission band with greatly reduced emission outside the emission band. The very small characteristic dimension $(5-10 \mu \mathrm{m})$ of the emitter results in low emittance outside the emission band. A new thin film rare earth-YAG (ytterium

${ }^{+}$NASA Resident Research Associate at Lewis Research Center 
aluminum garnet) emitter(5) shows great promise. The emittance theory presented in this paper applies to any thin film emitter. However, the emitter efficiency results are calculated specifically for the rare earths since a three band model is used in those calculations. In this model the spectrum is split into three wavelength regions; below emission band radiation which is described by a constant extinction coefficient, $\alpha_{w}$, within the emission band where $\alpha_{\lambda}$ depends on wavelength, $\lambda$, and above the emission band which is described by a constant extinction coefficient, $\alpha_{1}$

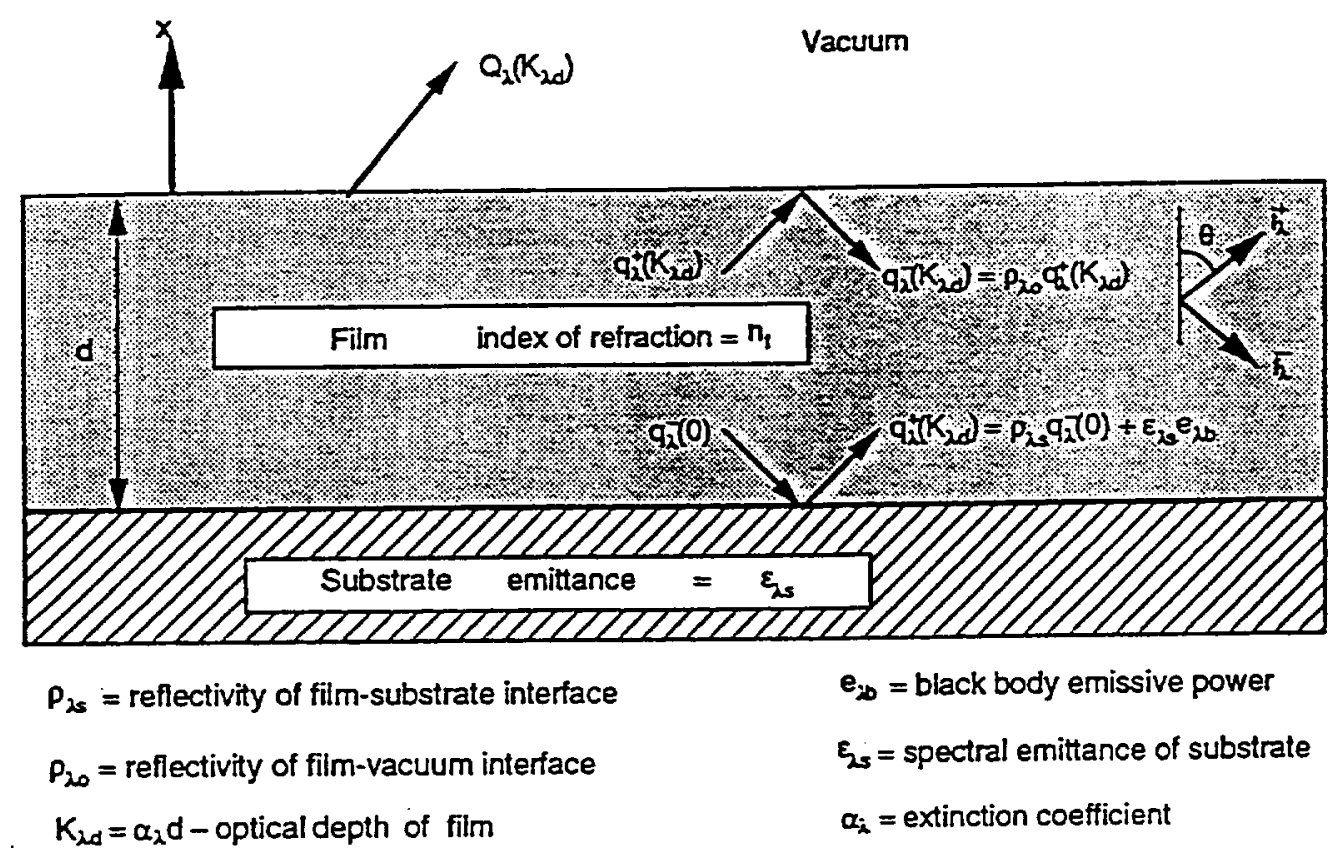

FIGURE 1. Schematic of Emitting Film

\section{RADIATTVE TRANSFER THEORY}

The thin film is approximated by the one dimensional model shown in figure 1 where thermal energy enters through the film substrate. All the film variables such as radiation intensity, $i_{\lambda}$, are assumed to vary only in the $x$ direction. This is an excellent approximation for thin films where the film dimensions perpendicular to the $\mathrm{x}$ direction are much larger than the film thickness, $\mathrm{d}$. The steady state one dimensional energy equation ${ }^{6}$ for the solid state film is 
$\frac{d}{d x}\left[k_{t} \frac{d T_{E}}{d x}\right]-\frac{d Q}{d x}=0$

where $T_{E}$ is the film temperature, $k_{t}$ is the film thermal conductivity and $Q$ is the total radiation energy flux.

$$
\begin{gathered}
Q=\int_{0}^{\infty} q_{\lambda} d \lambda \\
q_{\lambda}=\int_{\omega=4 \pi} i_{\lambda} \cos \theta d \theta
\end{gathered}
$$

The spectral intensity, $i_{\lambda}$, in terms of the wavelength, $\lambda$, is determined by the radiative transfer equation(6),

$$
\frac{1}{\alpha_{\lambda}} \frac{\mathrm{di}_{\lambda}}{\mathrm{dr}}+\mathrm{i}_{\lambda}=S_{\lambda}
$$

which gives the variation of the intensity in some direction, $r$, in terms of the socalled source function, $S_{\lambda}$ and the extinction coefficient, $\alpha_{\lambda}$.

$$
\alpha_{\lambda}=a_{\lambda}+\sigma_{\lambda}
$$

where $a_{\lambda}$ is the absorption coefficient and $\sigma_{\lambda}$ is the scattering coefficient. The source function includes both emission and scattering and depends on the scattering albedo defined as

$$
\Omega_{\lambda}=\frac{\sigma_{\lambda}}{\sigma_{\lambda}+\alpha_{\lambda}}=\frac{\sigma_{\lambda}}{\alpha_{\lambda}}
$$

In this analysis we assume that $T_{E}$ is constant throughout the film although experimental results for the rare earth-YAG thin film emitter(5) show a temperature gradient across the emitter $\left(200-300^{\circ} \mathrm{K}\right)$. An analysis that includes temperature variation is reported in another paper ${ }^{8}$ at this meeting. With $T_{E}=$ constant, the limiting solution to eq. (1) is $q=$ constant and all the thermal input leaves the film as radiation. The details of the solution for the source function, 
$S_{\lambda}$, and intensity, $i_{\lambda}$, are given in ref. 7 (equations are in terms of frequency, $v$,rather than wavelength, $\lambda$ ) To solve for $i_{\lambda}$ and $S_{\lambda}$ the following approximations were made. (1) The temperature, $T_{E}$, is uniform so that $a_{\lambda}, \sigma_{\lambda}$, and film index of refraction, $n_{\lambda}$, are also constant. (2) The film is a pure dielectric; therefore $n_{f}$ is a real number. (3) Boundaries at $x=0$ and $x=d$ behave diffusively; therefore $i_{\lambda}(0)$, $i_{\lambda}(d)$, reflectivity, $\rho_{\lambda_{s}}$, and emittance, $\varepsilon_{\lambda s}$ at the substrate-film interface and reflectivity at the upper boundary, $\rho_{\lambda_{0}}$ are independent of $\theta$. (4) Scattering is isotropic. (5) Interference effects are neglected.

In solving the one dimensional radiative transfer equation (eq. (4)) it is convenient to split the intensity into two parts; $i_{\lambda}+$ for radiation in the positive $x$ direction $(0 \leq \theta$ $\leq \pi / 2)$ and $i_{\lambda}{ }^{-}$for radiation in the negative $x$ direction $(\pi / 2 \leq \theta \leq \pi)$. Using the definitions of $i_{\lambda}{ }^{+}$and $i_{\lambda}{ }^{-}$and assumption (3) the boundary conditions at $x=0$ and $\mathrm{x}=\mathrm{d}$ are the following.

$$
\begin{gathered}
\mathrm{q}_{\lambda}^{+}(0)=2 \pi \int_{0}^{\frac{\pi}{2}} \mathrm{i}_{\lambda}^{+}(0) \cos \theta \sin \theta \mathrm{d} \theta=\pi \mathrm{i}_{\lambda}^{+}(0)=\varepsilon_{\lambda_{s}} \mathrm{e}_{\lambda b}+\rho_{\lambda_{s}} \mathrm{q}_{\lambda}^{-}(0) \\
\mathrm{q}_{\lambda}^{-}(\mathrm{d})=\pi \mathrm{i}_{\lambda}^{-}(\mathrm{d})=\pi \rho_{\lambda_{0}} \mathrm{i}_{\lambda}^{+}(\mathrm{d})
\end{gathered}
$$

Appearing in eq. (6) is the black body emissive power,

$$
e_{\lambda b}(\lambda, T)=\pi i_{\lambda b}=\frac{2 \pi h c_{o}^{2}}{\lambda^{5}\left[\exp \left(h c_{d} \lambda k T\right)-1\right]}
$$

where $h$ is Plank's constant, $k$ is Boltzmann's constant, and $c_{o}$ is the vacuum speed of light.

Defining the optical depth,

$$
\mathrm{K}_{\lambda}=\int_{0}^{x} \alpha_{\lambda} \mathrm{dx} x^{\prime}=\alpha_{\lambda} \mathrm{x}
$$

equation (4) can be integrated to obtain $i_{\lambda}{ }^{+}\left(K_{\lambda d}\right)$ and $i_{\lambda}{ }^{-}(0)$, where 


$$
K_{\lambda d}=\alpha_{\lambda} d
$$

and those results together with the boundary conditions can be used to calculate the radiation fluxes(7) at the boundaries.

$$
\begin{aligned}
\mathrm{q}_{\lambda}^{+}\left(\mathrm{K}_{\lambda_{d}}\right) & =2 \pi \int_{0}^{\frac{\pi}{2}} \mathrm{i}_{\lambda}^{+}\left(\mathrm{K}_{\lambda_{d}}, \theta\right) \cos \theta \sin \theta d \theta \\
& =2\left[\varepsilon_{\lambda_{s}} \mathrm{e}_{\lambda \mathrm{b}}+\rho_{\lambda_{s}} \mathrm{q}_{\lambda}^{-}(0)\right] \mathrm{E}_{3}\left(\mathrm{~K}_{\lambda_{d}}\right)+\Phi_{+} \\
\mathrm{q}_{\bar{\lambda}}^{-}(0) & =-2 \pi \int_{\frac{\pi}{2}}^{\pi} \bar{i}_{\lambda}^{-}(0) \cos \theta \sin \theta d \theta \\
& =2 \rho_{\lambda_{0}} \mathrm{q}_{\lambda}^{+}\left(\mathrm{K}_{\lambda_{d}}\right) \mathrm{E}_{3}\left(\mathrm{~K}_{\lambda_{d}}\right)+\Phi_{-}
\end{aligned}
$$

Solving equations (12) and (13) results in the following

$$
\begin{gathered}
\mathrm{q}_{\lambda}^{+}\left(\mathrm{K}_{\lambda d}\right)=\frac{2\left[\varepsilon_{\lambda_{s}} \mathrm{e}_{\lambda_{b}}+\rho_{\lambda_{s}} \Phi_{]}\right] \mathrm{E}_{3}\left(\mathrm{~K}_{\lambda_{d}}\right)+\Phi_{+}}{1-4 \rho_{\lambda_{s}} \rho_{\lambda_{0}} \mathrm{E}_{3}^{2}\left(\mathrm{~K}_{\lambda_{d}}\right)} \\
\mathrm{q}_{\lambda}^{-}(0)=\frac{2 \rho_{\lambda_{0}}\left[\Phi_{+}+2 \mathrm{E}_{3}\left(\mathrm{~K}_{\lambda_{d}}\right) \varepsilon_{\lambda_{s}} e_{\lambda_{b}}\right] \mathrm{E}_{3}\left(\mathrm{~K}_{\lambda_{d}}\right)+\Phi_{-}}{1-4 \rho_{\lambda_{s}} \rho_{\lambda_{0}} \mathrm{E}_{3}^{2}\left(\mathrm{~K}_{\lambda_{d}}\right)}
\end{gathered}
$$

where,

$$
\begin{aligned}
& \Phi_{+}=2 \pi \int_{0}^{\mathrm{K}_{\lambda d}} \mathrm{~S}_{\lambda}\left(\mathrm{K}_{\lambda}^{*}\right) \mathrm{E}_{2}\left(\mathrm{~K}_{\lambda d}-\mathrm{K}_{\lambda}^{*}\right) \mathrm{d} \mathrm{K}_{\lambda}^{*} \\
& \Phi_{-}=2 \pi \int_{0}^{\mathrm{K}_{\lambda d}} \mathrm{~S}_{\lambda}\left(\mathrm{K}_{\lambda}^{*}\right) \mathrm{E}_{2}\left(\mathrm{~K}_{\lambda}^{*}\right) \mathrm{d} \mathrm{K}_{\lambda}^{*}
\end{aligned}
$$

and the exponential integral, $E_{n}(u)$, is 


$$
E_{n}(u)=\int_{0}^{1} v^{n-2} \exp \left(-\frac{u}{v}\right) d v
$$

Once the source function, $S_{\lambda}$, is known, the boundary fluxes $q_{\lambda}{ }^{+}\left(K_{\lambda}\right)$ and $q_{\lambda}{ }^{-}(0)$ can be calculated and thus the hemispherical spectral emittance as well. The emittance is defined as

$$
\varepsilon_{\lambda} \equiv \frac{Q_{\lambda}\left(K_{\lambda d}\right)}{e_{\lambda b}\left(\lambda, T_{E}\right)}
$$

where $Q_{\lambda}\left(K_{\lambda d}\right)$ is the spectral emissive power at $K_{\lambda}=K_{\lambda d}$. To include the refractive properties of the boundary at $x=d$ the maximum angle, $\theta_{M}$, that allows radiation to escape the film is given by Snell's law. For $x>d$ we assume $n_{0}=1$, therefore Snell's law yields the following result.

$$
\mu_{M}^{2} \equiv \cos ^{2} \theta_{M}=1-\left(n_{\nu}\right)^{-2}
$$

For $\theta>\theta_{M}$ radiation is totally reflected. Including angles greater than $\theta_{M}$ would allow the possibility of $\varepsilon_{\lambda}>1(6)$. Therefore, using the boundary condition given by eq. (8) the emissive power, $Q_{\lambda}$, is the following

$$
\begin{aligned}
Q_{\lambda}\left(K_{\lambda d}\right) & =2 \pi\left[\int_{0}^{\theta_{M}} i_{\lambda}^{+}\left(K_{\lambda d}\right) \cos \theta \sin \theta d \theta-\rho_{\lambda_{0}} \int_{0}^{\theta_{M}} i_{1}^{+}\left(K_{\lambda d}\right) \cos \theta \sin \theta d \theta\right] \\
& =\left(1-\rho_{\lambda_{0}}\right)\left\{q_{\lambda}^{+}\left(K_{\lambda d}\right)-2 \mu_{M}^{2}\left[E_{\lambda_{s}} e_{\lambda b}+\rho_{\lambda_{s}} q_{\lambda}^{-}(0)\right] E_{3}\left(\frac{K_{\lambda d}}{\mu_{M}}\right)-\Phi_{M}\right\}
\end{aligned}
$$

where

$$
\Phi_{M}=2 \pi \mu_{M} \int_{0}^{K_{\lambda d}} S_{\lambda}\left(K_{\lambda}^{*}\right) E_{2}\left(\frac{K_{\lambda d}-K_{\lambda}^{*}}{\mu_{M}}\right) d K_{\lambda}^{*}
$$

In reference 7 the source function is obtained for isotropic scattering and diffuse boundaries (assumptions (3) and (4)). The solution is obtained in the following 
manner. First, the exponential integral functions in the source function equation are approximated by simple exponential functions. This allows the integral source function equation to be reduced to a linear second order differential equation which can then be solved. With no scattering the source function has the following simple form.

$$
S_{\lambda}=n_{\lambda}^{2} i_{\lambda b}\left(\lambda, T_{E}\right) \quad \sigma_{\lambda}=0
$$

Since we have assumed $T_{E}$ is uniform, $S_{\lambda}$ has no $K_{\lambda}$ dependence and the integrations appearing in $\Phi_{+}, \Phi_{-}$and $\Phi_{M}$ can be carried out. Also, since the substrate is opaque

$$
\rho_{\lambda s}=1-\varepsilon_{\lambda s}
$$

Therefore using equations (14), (15) and (24) in equation (21) yields the following result for the spectral emittance

$\varepsilon_{\lambda}=\frac{\left(1-\rho_{\lambda \sigma}\right)}{D_{\lambda}}\left\{2 h_{1}\left[\varepsilon_{\lambda s}+n_{\lambda}^{2}\left(1-\varepsilon_{\lambda s}\right)\left(1-2 E_{3}\left(K_{\lambda d}\right)\right]+n_{\lambda}^{2}\left[h_{+}\left(1-2 E_{3}\left(K_{\lambda d}\right)\right)-\mu_{M}^{2} D_{\lambda}\left(1-2 E_{3}\left(\frac{K_{\lambda d}}{\mu_{M}}\right)\right)\right]\right.\right.$

where,

$$
\begin{aligned}
& \mathrm{h}_{+}=1-4 \rho_{\lambda s} \rho_{\lambda_{0}} \mu_{M}^{2} \mathrm{E}_{3}\left(\mathrm{~K}_{\lambda d}\right) \mathrm{E}_{3}\left(\frac{\mathrm{K}_{\lambda d}}{\mu_{M}}\right) \\
& \mathrm{h}_{-}=\mathrm{E}_{3}\left(\mathrm{~K}_{\lambda_{d}}\right)-\mu_{\mathrm{M}}^{2} \mathrm{E}_{3}\left(\frac{\mathrm{K}_{\lambda d}}{\mu_{\mathrm{M}}}\right) \\
& \mathrm{D}_{\lambda}=1-4 \rho_{\lambda s} \rho_{\lambda_{0}} \mathrm{E}_{3}^{2}\left(\mathrm{~K}_{\lambda d}\right)
\end{aligned}
$$

At the emitting surface $(x=d)$ we assume that the reflectance, $\rho_{\lambda_{0}}$, can be approximated by the normal reflectance for a specular reflective dielectric interface. Therefore, assuming $n_{o}=1.0$ for $x>d$ the reflectance is the following(6)

$$
\rho_{\lambda_{0}}=\left(\frac{n_{\lambda}-1}{n_{\lambda}-1}\right)^{2}
$$


Equation (25) shows the exponential dependence $\left[E_{3}(x) \approx 1 / 2 \mathrm{e}^{(-3 / 2 x)}\right]$ of $\varepsilon_{\lambda}$ on the optical depth, $\mathrm{K}_{\lambda_{\mathrm{d}}}$. Also, the importance of substrate emittance is apparent. For $\lambda$ regions outside the emission band where $K_{\lambda d}$ is small $\left[E_{3}(0)=1 / 2\right]$ the emittance will be dominated by the substrate contribution. Thus it is important to have low substrate emittance to minimize the out of band emission and thus maximize the emitter efficiency. Low $\varepsilon_{\lambda_{s}}$ also implies $\rho_{\lambda s}$ approaches 1 which means that a significant contribution ( $1-\varepsilon_{\lambda s}$ term in eq. (25)) to the emission band results from reflection at the substrate. Therefore, low substrate emittance minimizes out of band radiation and increases within band radiation.

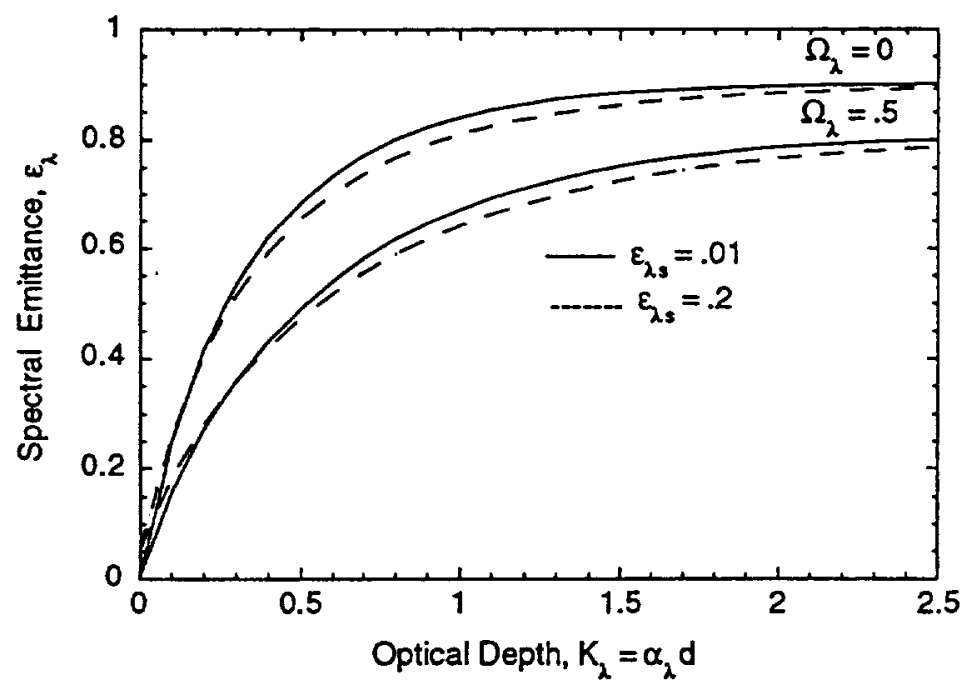

FIGURE 2. Thin film spectral emittance as a function of optical depth for scattering albedo $=0, .5$ and substrate emittance $=.01, .2$. Also, film index of refraction $=1.9$, reflectance at film-substrate interface $=1-\varepsilon$.

In figure 2 the spectral emittance is shown as a function of the optical depth for two substrate emittances $\left(\varepsilon_{\lambda s}=.01, .2\right)$ and $\mathrm{n}_{\lambda}=1.9$, which is representative of rare earth emitters. Results for both no scattering $\left(\sigma_{\lambda}=0\right)$ and equal scattering and absorption coefficients $\left(\sigma_{\lambda}=\alpha_{\lambda}, \Omega_{\lambda}=.5\right)$ are shown. The $\Omega_{\lambda}=.5$ results were obtained from reference 7 . Several observations can be made from the results in fig. 2. First, scattering obviously reduces the spectral emittance however, emitter efficiency will not be greatly affected since the ratio of the emittance within the emission band to the emittance outside the emission band determines the emitter efficiency. This will be discussed later.. The second thing to notice is how the substrate emittance affects the spectral emittance. Although it is difficult to see in the figure, for $\mathrm{K}_{\lambda d} \rightarrow 0$ the spectral emittance increases with increasing substrate emittance.However,as $\mathrm{K}_{\lambda d}$ increases the opposite result occurs; the spectral emittance decreases as substrate emittance increases. This result occurs because of the reflectance term, $\left(1-\varepsilon_{\lambda s}\right)$, in the spectral emittance (eq. (25)). For $\mathrm{K}_{\lambda_{d}} \rightarrow 0$ the 
reflectance term is negligible. But as $\mathrm{K}_{\lambda d}$ increases the reflectance term becomes important so that increasing reflectance (decreasing substrate emittance) results in increased spectral emittance. Thus, as already mentioned, low substrate emittance is important for two reasons. For emission outside the emission band where $\mathrm{K}_{\lambda d}$ is small, low substrate emittance is required to minimize the emission. Within the emission band where $K_{\lambda d}$ is larger, low substrate emittance results in high reflectance at the substrate and thus increases the emission.

\section{EMITTER EFFICIENCY}

As stated earlier, the emission properties of the rare earth emitters can be approximated by a three band model and the emitter efficiency is defined as

$$
\eta_{E} \equiv \frac{Q_{b}}{Q_{1}+Q_{b}+Q_{u}}
$$

and the power in each of the three bands is defined by

$$
\begin{aligned}
& Q_{b}=\int_{\lambda_{u}}^{\lambda_{1}} \varepsilon_{\lambda} e_{\lambda b} d \lambda \\
& Q_{1}=\int_{\lambda_{1}}^{-} \varepsilon_{\lambda} e_{\lambda_{b}} \mathrm{~d} \lambda \\
& Q_{u}=\int_{0}^{\lambda_{u}} \varepsilon_{\lambda} e_{\lambda_{b}} \mathrm{~d} \lambda
\end{aligned}
$$

For the below and above band regions we assume a constant extinction coefficient, as well as, constant substrate emittance and film index of refraction so that the emittance is a constant in those bands.

$$
\begin{array}{lll}
\varepsilon_{1}=\varepsilon_{\lambda}\left(\mathrm{K}_{1}, \varepsilon_{1 s}, n_{1}\right) & \lambda_{1} \leq \lambda \leq \infty, & \mathrm{K}_{1}=\alpha_{1} \mathrm{~d} \\
\varepsilon_{\mathrm{u}}=\varepsilon_{\lambda}\left(\mathrm{K}_{\mathrm{u}}, \varepsilon_{\mathrm{us}}, \mathrm{n}_{\mathrm{u}}\right) & 0 \leq \lambda \leq \lambda_{\mathrm{u}}, & \mathrm{K}_{\mathrm{u}}=\alpha_{\mathrm{u}} \mathrm{d}
\end{array}
$$

Here $\varepsilon_{1 s}$ and $\varepsilon_{u s}$ are the substrate emittances in the above and below band regions 
$\mathrm{n}_{1}$ and $\mathrm{n}_{\mathrm{u}}$ are the film indexes of refraction in those bands. For the emission band the extinction coefficient varies considerably for the rare-earth garnet emitters(5). Therefore, $\varepsilon_{\lambda}$ will be a function of wavelength within the emission band. Since $\varepsilon_{u}$ and $\varepsilon_{1}$ are independent of $\lambda$, the integrations in equations (31b) and (31c) can be performed so that the following is obtained for $\eta_{E}$

$$
\eta_{E}=\left[1+\frac{\varepsilon_{1} G\left(u_{1}\right)+\varepsilon_{u} H\left(u_{u}\right)}{F\left(\lambda_{1}, \lambda_{u}, T_{E}\right)}\right]^{-1}
$$

where,

$$
\begin{gathered}
G\left(u_{1}\right)=\frac{\pi^{4}}{15}-\int_{u_{1}}^{\infty} \frac{x^{3}}{e^{x}-1} d x \quad u_{1}=\frac{h c_{o}}{\lambda_{1} k T_{E}} \\
H\left(u_{u}\right)=\int_{u_{0}}^{\infty} \frac{x^{3}}{e^{x}-1} d x \quad u_{u}=\frac{h c_{o}}{\lambda_{u} k T_{E}} \\
F\left(\lambda_{1}, \lambda_{u}, T_{E}\right)=\lambda_{b}^{4} \int_{\lambda_{b}}^{\lambda_{1}} \varepsilon_{\lambda}\left(K_{\lambda}, \varepsilon_{\lambda_{s}}, n_{\lambda_{b}}\right) \frac{d \lambda}{\lambda^{5}\left[\exp \left(h c_{d} \lambda k T_{E}-1\right]\right.} \quad \lambda_{b}=\frac{h c_{o}}{k T_{E}}
\end{gathered}
$$

The emitter efficiency for the thin film rare earth-YAG emitters was calculated using eq. (33). For no scattering the emittance is given by equation (25). With scattering the emittance was obtained from ref. 7. The extinction coefficients for Er-YAG and Ho-YAG were obtained from ref. 5 . From the experimental extinction coefficient data the emission band is defined by $\lambda_{1}=1.66 \mu \mathrm{m}\left(\alpha_{1}=.181 \mathrm{~cm}^{-1}\right), \lambda_{\mathrm{u}}=1.42 \mu \mathrm{m}$ $\left(\alpha_{\mathrm{u}}=.397 \mathrm{~cm}^{-1}\right)$ for Er-YAG and by $\lambda_{1}=2.15 \mu \mathrm{m}\left(\alpha_{1}=.056 \mathrm{~cm}^{-1}\right) \lambda_{\mathrm{u}}=1.82 \mu \mathrm{m}$ $\left(\alpha_{\mathrm{u}}=.611 \mathrm{~cm}\right)$ for $\mathrm{Ho}-\mathrm{YAG}$. 


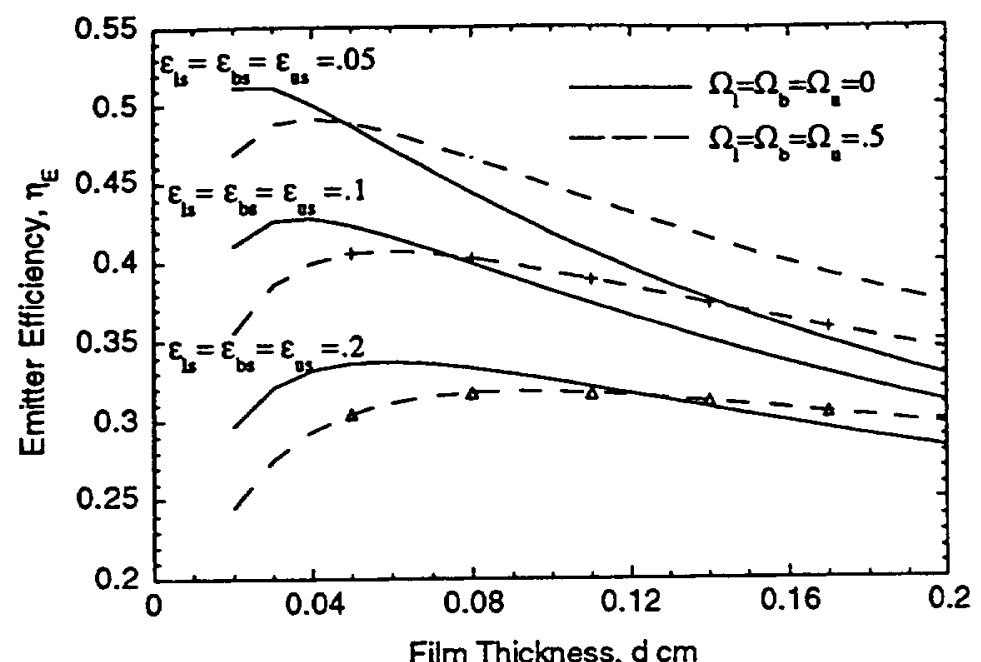

3a) $\operatorname{Er}(40 \%)-Y A G\left(\lambda_{\mathrm{u}}=1.42 \mu \mathrm{m}, \alpha_{\mathrm{u}}=.397 \mathrm{~cm}^{-1}, \lambda_{1}=1.66 \mathrm{~mm}, \alpha_{1}=.181 \mathrm{~cm}^{-1}\right)$

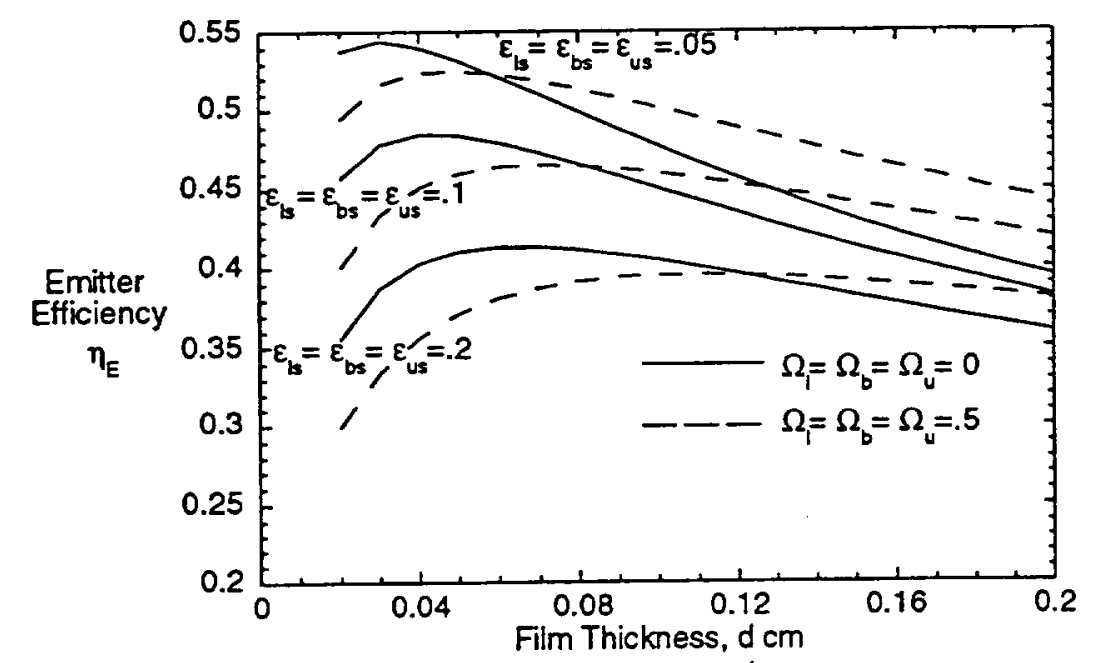

3b) $\mathrm{Ho}(25 \%)-Y A G\left(\lambda_{u}=1.82 \mu \mathrm{m}, \alpha_{\mathrm{u}}=.611 \mathrm{~cm}^{-1}, \lambda_{1}=2.15 \mu \mathrm{m}, \alpha_{1}=.056 \mathrm{~cm}^{-1}\right)$

FIGURE 3. Dependence of Er-YAG and HO-YAG emitter efficiency, $\eta_{E}$, on scatterig albedo, $\Omega$, and substrate emittance, $\varepsilon_{z}$, for $T_{E}=1500 \mathrm{~K}$, (index of refraction $=1.9$ )

Figure 3 shows the efficiency for Er-YAG and Ho-YAG as a function of film thickness, $d$, at $T_{E}=1500 \mathrm{~K}$ for no scattering and for equal absorption and scattering coefficients $(\Omega=.5$ ). The substrate emittance and scattering albedo were assumed to be constants $\left(\varepsilon_{\mathrm{s}}=\varepsilon_{\mathrm{is}}=\varepsilon_{\mathrm{bs}}=\varepsilon_{\mathrm{us}} ; \Omega=\Omega_{1}=\Omega_{\mathrm{b}}=\Omega_{\mathrm{v}}\right)$. Also, the refractive index was assumed to be constant $\left(\mathrm{n}_{\mathrm{fl}}=\mathrm{n}_{\mathrm{fb}}=\mathrm{n}_{\mathrm{fu}}=1.9\right)$. The value $\mathrm{n}_{\mathrm{f}}=$ 1.9 is representative of rare earth oxides(9). Several observations can be made from the results shown in figure 3 . First, there is an optimum film thickness for maximum efficiency. Second, scattering causes only a small reduction in $\eta_{\mathrm{E}}$ - 
However, a thicker film is required to obtain maximum $\eta_{E}$ when scattering exists. As will be discussed later, scattering has a more significant effect on the emitted power. Finally, to have large $\eta_{E}$ the substrate must have low emittance.

Now consider the effect of emitter temperature, $T_{E}$, on the emitter efficiency. In figure $4 \eta_{E}$ for Er-YAG and Ho-YAG is shown for $T_{E}=1200 \mathrm{~K}, 1500 \mathrm{~K}$, $1750 \mathrm{~K}$ and $2000 \mathrm{~K}$. The values for $\alpha_{1}, \alpha_{u}, \varepsilon_{s}$ and $n_{f}$ are the same as in figure 3 . For the scattering albedo, $\Omega_{1}=\Omega_{b}=\Omega_{u}=1 / 3\left(\sigma_{\lambda}=1 / 2 a_{\lambda}\right)$ was chosen as representative of the rare earth-YAG emitter. Figure 4 shows the strong temperature dependence of $\eta_{E}$. Two observations can be made. First of all the efficiency increases rapidly with temperature until a maximum value is reached and then decreases. In the case of Ho-YAG maximum efficiency occurs for $T_{E}=1600 \mathrm{~K}$. For Er-YAG maximum efficiency occurs for $T_{E}>2000 \mathrm{~K}$. In references 7 and 10 , where the emission band is characterized by a single constant extinction coefficient, maximum efficiency was found to occur for $E b / k T_{E} \approx 4$, where $E_{b}=h c_{o} / \lambda_{b}$. In the case of $\operatorname{Er}\left(\lambda_{b}=1.54 \mu \mathrm{m}\right)$ this corresponds to $T_{E} \approx 2400 \mathrm{~K}$ and for Ho $\left(\lambda_{b}=1.99\right.$ $\mu \mathrm{m}) \mathrm{T}_{E} \approx 1800 \mathrm{~K}$. Second, the thickness for maximum efficiency decreases as the temperature increases. For Er-YAG the effect is small. However, for Ho-YAG the change is significant. At $T_{E}=1200 \mathrm{~K}$ the optimum thickness is approximately 0.1 $\mathrm{cm}$ but at $\mathrm{T}_{\mathrm{E}}=2000 \mathrm{~K}$ the optimum thickness is approximately $0.05 \mathrm{~cm}$. The difference in the extinction coefficient within the emission band accounts for the different behavior between Er-YAG and Ho-YAG. The magnitude of $\alpha_{\lambda}$ for ErYAG is approximately a factor of 2 larger than $\alpha_{\lambda}$ for Ho-YAG ${ }^{5}$ within the emission bands. Also, there is a difference in the shape of the $\alpha_{\lambda}$ vs $\lambda$ curves for Er-YAG and Ho-YAG(5). Both materials have two peaks in the $\alpha_{\lambda}$ vs $\lambda$ curves. However, for Ho-YAG the peaks are separated by a greater wavelength difference. As a result the optimum thicknesses for Er-YAG are smaller than optimium thicknesses for Ho-YAG. 


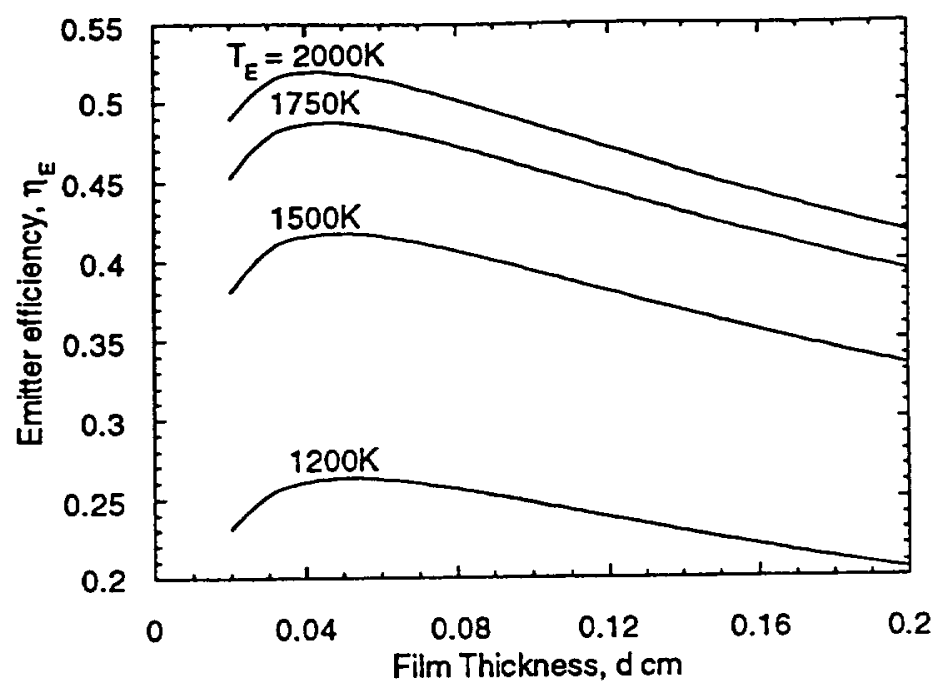

4a) $\operatorname{Er}(40 \%)$-YAG $\left(\lambda_{u}=1.441 \mathrm{~m}, \alpha_{u}=.397 \mathrm{~cm}^{-1}, \lambda_{1}=1.66 \mu \mathrm{m}, \alpha_{1}=.181 \mathrm{~cm}^{-1}\right)$

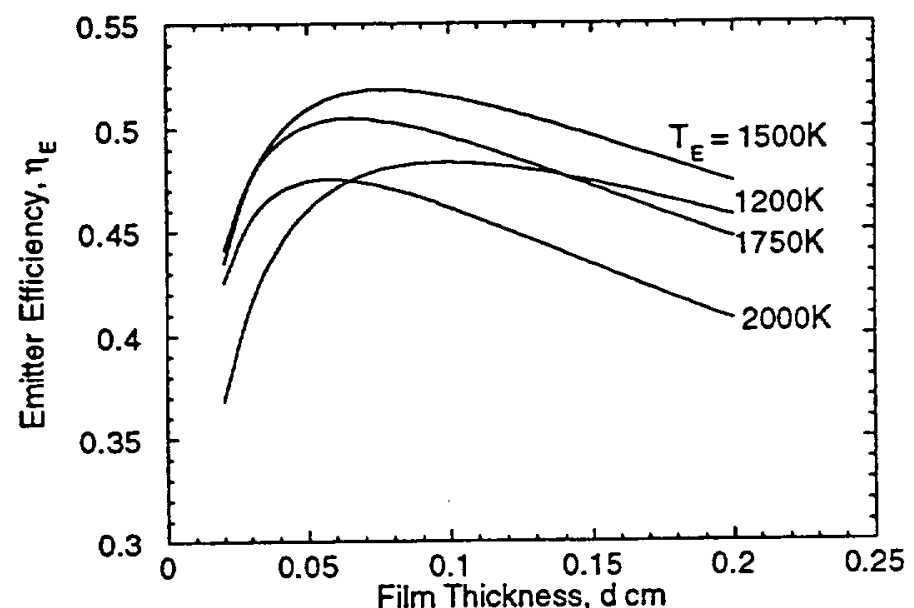

4b) $H \circ(25 \%)-$ YAG $\left(\lambda_{u}=1.82, \alpha_{u}=.611 \mathrm{~cm}^{-1}, \lambda_{1}=2.15 \mu \mathrm{m}, \alpha_{1}=.056 \mathrm{~cm}^{-1}\right)$ FIGURE 4. Emitter Efficiency of Er-YAG and Ho-YAG as a function of film thickness for various temperatures. Scattering albedo $(\Omega=1 / 3)$, substrate emittance $\left(e_{3}=1\right)$, and index of refraction $(n=1.9)$, the same in all bands. 


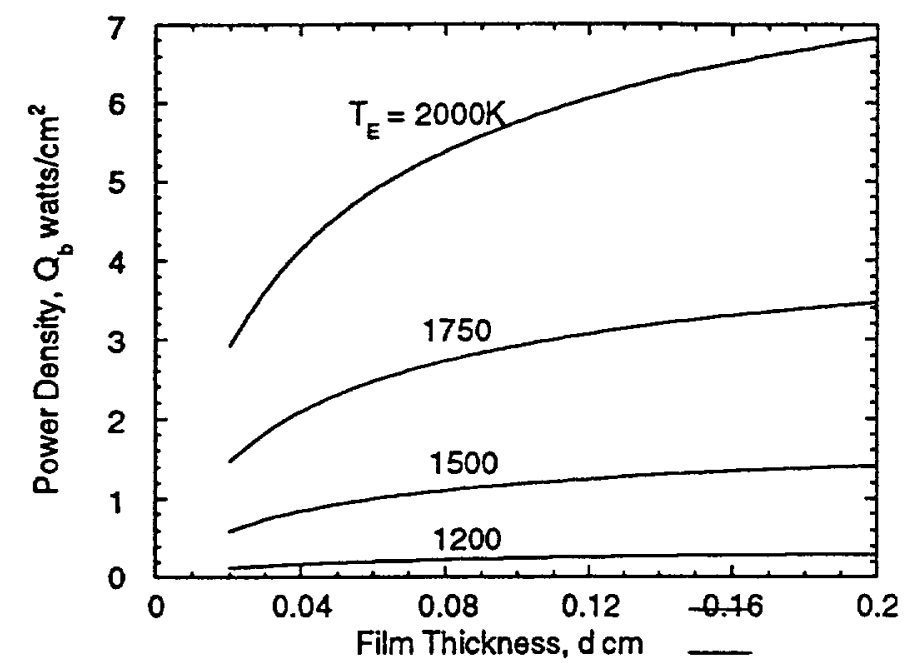

5a) $\operatorname{Er}(40 \%)-$ YAG $\left(\lambda_{v}=1.42 \mu \mathrm{m}, \alpha_{u}=.397 \mathrm{~cm}^{-1}, \lambda_{1}=1.66 \mu \mathrm{m}, \alpha_{1}=.181 \mathrm{~cm}^{-1}\right)$

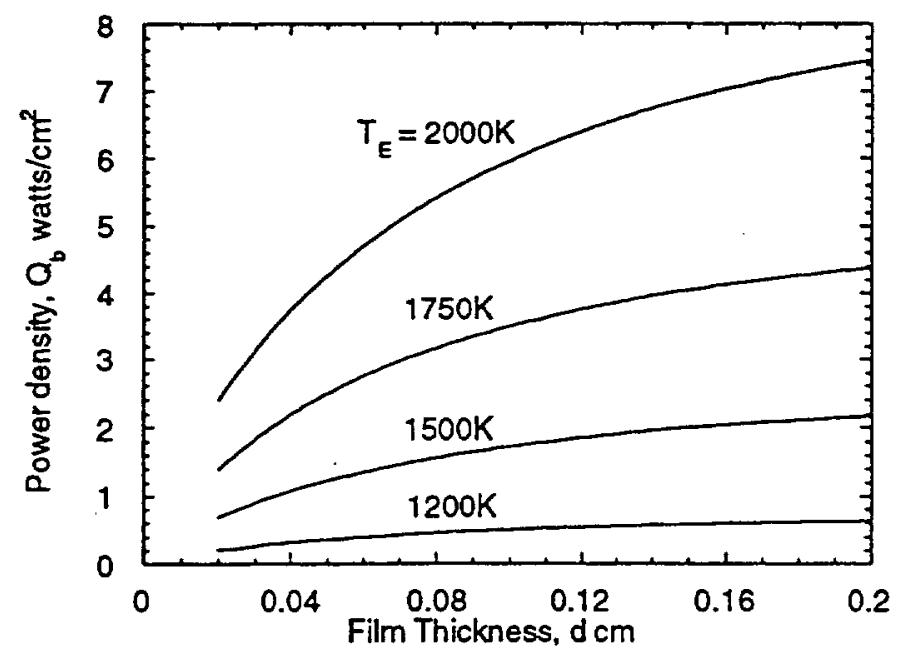

5b) $\mathrm{Ho}(25 \%)$ - YAG $\left(\lambda_{\mathrm{u}}=1.82 \mu \mathrm{m}, \alpha_{\mathrm{u}}=.611 \mathrm{~cm}^{-1}, \lambda_{1}=2.15 \mu \mathrm{m}, \alpha_{1}=.056 \mathrm{~cm}^{-1}\right)$

FIGURE 5. Power density as a function of film thickness for various emitter temperatures. Scattering albedo $(\Omega=1 / 3)$, substrate emittance $\left(\varepsilon_{s}=1\right)$,

and index of refraction $(n=1.9)$ the same in all three bands.

\section{EMITTER POWER DENSITY}

Besides efficiency, the other important emitter performance parameter is the emitted power in the emission band, $Q_{b}$. The efficiency is strongly dependent on the emittance ratios, $\varepsilon_{\mathrm{g}} / \varepsilon_{\mathrm{b}}$ and $\varepsilon_{\mathrm{u}} / \varepsilon_{\mathrm{b}}$, where $\varepsilon_{\mathrm{b}}$ is the emittance within the emission band. Whereas, efficiency is not strongly dependent on the magnitude of the emittance in the three bands (see eq. (33) and ref. 10). However, $Q_{b}$ is directly 
proportional to the magnitude of the emittance within the emission band. Therefore, if the magnitude of the emission band emittance, $\varepsilon_{b}$, is low, the emitted power, $Q_{b}$, will be low. However, the efficiency can still be high if $\varepsilon_{\mathrm{l}} / \varepsilon_{\mathrm{b}}$ and $\varepsilon_{\mathrm{l}} / \varepsilon_{\mathrm{b}}$ are small. The efficiency and power density are not strongly coupled. It is possible to have both high efficiency with low emitted power and low efficiency with high emitted power.

In figure 5 the emitted power density, $Q_{b}$, is shown as a function of film thickness for Er-YAG and Ho-YAG for the same conditions as figure 4. As can be seen, $Q_{b}$ increases monotonically with $d$. This results because $\varepsilon_{b}$ increases monotonically with $d$ (see fig. 2). Thus by increasing thickness, emitted power increases, but efficiency will begin to decrease when the thickness exceeds the thickness for maximum efficiency. Also, since increasing scattering reduces $\varepsilon_{\lambda}$ (see fig. 2), $Q_{b}$ will also be reduced by increased scattering(7). Note that $Q_{b}$ for HoYAG is greater than $Q_{b}$ for Er-YAG for the temperatures $\left(T_{E} \leq 2000 \mathrm{~K}\right.$ ) shown in figure 5. If the temperature were increased beyond $2000 \mathrm{~K}, \mathrm{Q}_{b}$ for Er- YAG would eventually exceed $Q_{b}$ for Ho-YAG.

\section{CONCLUSION}

A radiative transfer analysis that includes isotropic scattering and assumes constant temperature throughout the film was used to calculate the spectral emittance, $\varepsilon_{\lambda}$, of a thin film. Using $\varepsilon_{\lambda}$ based on measured extinction coefficients and assuming a three band model for the rare earth-YAG thin film emitter, the emitter efficiency, $\eta_{E}$, and emitted power density, $Q_{b}$, were calculated. Efficiencies of approximately $50 \%$ are possible with power densities of several watts $/ \mathrm{cm}^{2}$ in the temperature range $1200 \leq T_{E} \leq 2000 \mathrm{~K}$ for Ho-YAG and Er- YAG. In order to attain high efficiency, low substrate emittance is required. Also, there is an optimum thickness in the range $.04 \leq \mathrm{d} \leq 0.1 \mathrm{~cm}$ to attain maximum efficiency. However, power density is a monotonically increasing function of film thickness, d. Finally, scattering has negligible effect on efficiency but a significant effect on power density.

\section{REFERENCES}

1. Dieke, G. H., Spectra and Energy Levels of Rare Earth Ions in Crystals, New York Interscience, 1968.

2. Guazzoni, G. E. Appl. Spectra, 26, 60, 1972.

3. Nelson, R. E., "Rare Earth Oxide TPV Emitters," Proceedings of the 32nd International Power Sources Symposium, (Electrochemical Society, Pennington, NJ, 1986), pg. 95.

4. Parent, C. R. and Nelson, R. E. "Thermophotovoltaic Energy Conversion with a Novel Rare 
Earth Oxide Emitter," Proceedings of the 21st Intersociety Energy Conversion Conference, (American Chemical Soc., Washington, DC, 1986), 2, 1314.

5. Lowe, R. A., Chubb, D. L., Farmer, S. C. and Good, B. S. Appl. Phys. Lett., 64, 3551 (1994).

6. Siegel, R. \& Howell, J. R., Thermal Radiation Heat Transfer, 2nd ed., Washington, DC, Hemisphere, 1981, Chap. 14, 19-6.2, p. 96.

7. Chubb, D. L. \& Lowe, R. A., J. Appl. Phys., 74, 5687 (1993).

8. Good, B. S., Chubb, D. L. and Lowe, R. A., "Temperature-Dependent Efficiency Calculations for a Thin-Film Selective Emitter," The First NREL Conference on Thermophotovoltaic Generation of Electricity, American Institute of Physics Series of Conference Proceedings, 1994. 9. Marcinow, T. \& Truszkowska, K., Appl. Opt. 20, 1755 (1981).

10. Chubb, D. L. "Reappraisal of Solid Selective Emitters," in Proceedings of the 21st Photovoltaic Specialists Conference, (IEEE, New York, 1990), pp. 1326-1342; also NASA TM$103290,1990$. 

Public reporting burden for this collection of information is estimated to average 1 hour per response, including the time for teviewing instructions, searching existing data sources, gathering and maintaining the data needed, and completing and reviewing the collection of intormation. Send comments regarding this burden estimate or any other aspect of this collection of information, including suggestions for reducing this burden, to Washington Headquarters Services. Directorate for Intormation Operations and Reports, 1215 Jetterso
Davis Highway, Suite 1204, Arlington, VA 22202-4302, and to the Office of Management and Budget, Paperwork Reduction Project (0704-0188), Washington, DC 20503.

\begin{tabular}{|l|c|c|}
\hline 1. AGENCY USE ONLY (Leave blank) & $\begin{array}{c}\text { 2. REPORT DATE } \\
\text { August } 1994\end{array}$ & $\begin{array}{r}\text { 3. REPORT TYPE AND DATES COVERED } \\
\text { Technical Memorandum }\end{array}$ \\
\hline
\end{tabular}

\section{TITLE AND SUBTITLE}

5. FUNDING NUMBERS

Emittance Theory for Thin Film Selective Emitter

6. AUTHOR(S)

WU-233-01-0A

Donald L. Chubb, Roland A. Lowe, and Brian S. Good

7. PERFORMING ORGANIZATION NAME(S) AND ADDRESS(ES)

8. PERFORMING ORGANIZATION REPORT NUMBER

National Aeronautics and Space Administration

Lewis Research Center

Cleveland, Ohio 44135-3191

E-9097

9. SPONSORING/MONITOAING AGENCY NAME(S) AND ADDRESS(ES)

10. SPONSORINGMONITORING AGENCY REPORT NUMBER

National Aeronautics and Space Administration

Washington, D.C. 20546-0001

NASA TM-106727

\section{SUPPLEMENTARY NOTES}

Prepared for the First Conference on Thermophotovoltaic Generation of Electricity sponsored by the National Renewable Energy Laboratory, Copper Mountain, Colorado, July 24-26, 1994. Donald L. Chubb and Brian S. Good, NASA Lewis Research Center; Roland A. Lowe, Kent State University, Kent, Ohio 44242, and NASA Resident Research Associate at Lewis Research Center. Responsible person, Donald L. Chubb, organization code 5410, (216) $433-2242$.

12a. DISTRIBUTIONUAVAILABILITY STATEMENT

12b. DISTRIBUTION CODE

Unclassified - Unlimited

Subject Category 44

\section{ABSTRACT (Maximum 200 words)}

Thin films of high temperature garnet materials such as yttrium aluminum garnet (YAG) doped with rare earths are currently being investigated as selective emitters. This paper presents a radiative transfer analysis of the thin film emitter. From this analysis the emitter efficiency and power density are calculated. Results based on measured extinction coefficients for erbium-YAG and holmium-YAG are presented. These results indicated that emitter efficiencies of $50 \%$ and power densities of several watts $/ \mathrm{cm} 2$ are attainable at moderate temperatures $(<1750 \mathrm{~K})$.

\section{SUBJECT TERMS}

Selective emitter; Thin film; Spectral emittance

15. NUMBER OF PAGES

\begin{tabular}{|c|c|}
\hline $\begin{array}{c}\text { 17. SECURITY CLASSIFICATION } \\
\text { OF REPORT } \\
\text { Unclassified }\end{array}$ & $\begin{array}{c}\text { 18. SECURITY CLASSIFICATION } \\
\text { OF THIS PAGE } \\
\text { Unclassified }\end{array}$ \\
\hline
\end{tabular}

19. SECURITY CLASSIFICATION OF ABSTRACT Unclassified
20. LIMITATION OF ABSTRACT 